\title{
Spin-One DKP Equation in the Presence of Coulomb and Harmonic Oscillator Interactions in $(1+3)$-Dimension
}

\author{
H. Hassanabadi, ${ }^{1}$ Z. Molaee, ${ }^{2}$ \\ M. Ghominejad, ${ }^{2}$ and S. Zarrinkamar ${ }^{3}$ \\ ${ }^{1}$ Physics Department, Shahrood University of Technology, P.O. Box 3619995161-316, Shahrood, Iran \\ ${ }^{2}$ Physics Department, Semnan University, Semnan, Iran \\ ${ }^{3}$ Department of Basic Sciences, Garmsar Branch, Islamic Azad University, Garmsar, Iran
}

Correspondence should be addressed to H. Hassanabadi, h.hasanabadi@shahroodut.ac.ir

Received 27 August 2012; Accepted 26 September 2012

Academic Editor: S. H. Dong

Copyright (C) 2012 H. Hassanabadi et al. This is an open access article distributed under the Creative Commons Attribution License, which permits unrestricted use, distribution, and reproduction in any medium, provided the original work is properly cited.

In this work, we study Duffin-Kemmer-Petiau equation in the presence of coulomb and harmonic oscillator potentials in $(1+3)$-dimension for spin-one particles and we obtain energy eigenvalues and corresponding eigenfunctions.

\section{Introduction}

The Duffin-Kemmer-Petiau (Duffin, 1938; Kemmer, 1938; Petiau, 1936) equation is a firstorder relativistic wave equation for spin-zero and one bosons [1-3]. It is similar with Dirac equation in which we replace the algebra of the gamma matrices by beta matrices. More recently, there have been a renewed interest in DKP equation; particularly it has been applied to QCD (large and short distances) by Gribov [4], to covariant Hamiltonian dynamics by Kanatchikov [5] and has been generalized to curve space-time by Red'kov [6] and Lunardi et al. [7]. In addition, the relativistic model of $\alpha$-nucleus elastic scattering where they have been treated by the formalism of the DKP theory [8] and covariant Hamiltonian [9] in the casual approach $[10,11]$ and there has been an increasing interest on the DKP oscillator [12-17]. Recently, many articles have been devoted to investigate DKP theory under different types of potential; hence, we can cite the following [18-29]. Since the wave function includes all the necessary information about considering systems, the energy eigenvalues and corresponding eigenfunctions between interaction systems in relativistic quantum mechanics 
and in nonrelativistic quantum mechanics are studied more efficiently in recent years. In this study, we have investigated DKP equation with coulomb and harmonic oscillator potentials in $(1+3)$-dimension.

\section{DKP Equation}

The DKP equation in free field is given by (in natural units $\hbar=c=1$ ) [1-3]

$$
\left(i \beta^{\mu} \partial_{\mu}-m_{0}\right) \Psi=0
$$

$\beta^{\mu}$ are the DKP matrices which are satisfied in this algebra:

$$
\beta^{\mu} \beta^{v} \beta^{\lambda}+\beta^{\lambda} \beta^{v} \beta^{\mu}=g^{\mu v} \beta^{\lambda}+g^{\lambda v} \beta^{\mu},
$$

where $g^{\mu v}=\operatorname{diag}(1,-1,-1,-1)$ and $\left(g^{\mu v}\right)^{2}=1$, being the metric tensor of Minkowski spacetime.

For the spin-one case, $\beta^{\mu}$ matrices are

$$
\begin{gathered}
\beta^{0}=\left(\begin{array}{cccc}
0 & \overline{0} & \overline{0} & \overline{0} \\
\overline{0}^{T} & 0 & I & 0 \\
\overline{0}^{T} & I & 0 & 0 \\
\overline{0}^{T} & 0 & 0 & 0
\end{array}\right), \\
\beta^{i}=\left(\begin{array}{cccc}
0 & \overline{0} & e_{i} & \overline{0} \\
\overline{0}^{T} & 0 & 0 & -i S_{i} \\
-e_{i}^{T} & 0 & 0 & 0 \\
\overline{0}^{T} & -i S_{i} & 0 & 0
\end{array}\right)
\end{gathered}
$$

with $S$ matrices being $3 \times 3$ ones, $\left(S_{i}\right)_{j k}=-i \varepsilon_{i j k}$ where $\varepsilon_{i j k}$ is $1,-1,0$ for an even permutation, an odd permutation, and repeated indices, respectively. $e_{i}$ Matrices are $1 \times 3,\left(e_{i}\right)_{1 j}=\delta_{i j}$, that is,

$$
e_{1}=(1,0,0), \quad e_{2}=(0,1,0), \quad e_{3}=(0,0,1)
$$

$I$ and 0 , respectively,represent unit and null $3 \times 3$ matrices and $\overline{0}$ s are $1 \times 3$ ones [30].

\section{DKP Equation in Three-Dimensional Space-Time}

Furthermore, for an elastic scattering, the interaction is [31]

$$
U=S(r)+P S_{\mu}(r)+\beta^{0} V(r)+\beta^{0} P V_{P}(r),
$$

where each term has a specific Lorentz character. Two Lorentz vectors may be written as $\beta^{\mu}$ and $P \beta^{\mu}$ by assuming rotational invariance and parity conservation. DKP matrices have three 
irreducible representations: one-dimension representation where is trivial, five-dimension representation that is for spin-zero particles, and ten-dimension representation that is for spin-one particles [1-3].

The DKP equation in the presence of interaction is written as

$$
\left(i \beta^{\mu} \partial_{\mu}-m_{0}-\beta^{0} P V(r)\right) \Psi=0
$$

As usual, a solution of the following form removes the time component

$$
\Psi(x, y, z, t)=\exp \left(-i E_{n, l} t\right) \psi_{n, l}(x, y, z)
$$

as the problem is considered in one spatial dimension, we consider one quantum number and write the wave function as

$$
\psi_{n, l}^{T}(x, y, z)=\left(\varphi_{n, l}^{(1)}, \varphi_{n, l}^{(2)}, \varphi_{n, l}^{(3)}, \varphi_{n, l}^{(4)}, \varphi_{n, l}^{(5)}, \varphi_{n, l}^{(6)}, \varphi_{n, l}^{(7)}, \varphi_{n, l}^{(8)}, \varphi_{n, l}^{(9)}, \varphi_{n, l}^{(10)}\right)^{T}
$$

So we choose,

$$
\varphi_{n, l}^{(1)}=i \phi_{n, l}, \quad \vec{F}=\left(\varphi_{n, l}^{(2)}, \varphi_{n, l}^{(3)}, \varphi_{n, l}^{(4)}\right), \quad \vec{G}=\left(\varphi_{n, l}^{(5)}, \varphi_{n, l}^{(6)}, \varphi_{n, l}^{(7)}\right), \quad \vec{H}=\left(\varphi_{n, l}^{(8)}, \varphi_{n, l}^{(9)}, \varphi_{n, l}^{(10)}\right)
$$

Substituting of the above relations in (3.6), we have determined ten coupled equations as follows

$$
\begin{gathered}
i\left(\frac{\partial F_{n, l}^{(2)}}{\partial x}-\frac{\partial F_{n, l}^{(1)}}{\partial y}\right)=m_{0} H_{n, l}^{(3)} \\
i\left(\frac{\partial F_{n, l}^{(1)}}{\partial z}-\frac{\partial F_{n, l}^{(3)}}{\partial x}\right)=m_{0} H_{n, l}^{(2)} \\
i\left(\frac{\partial F_{n, l}^{(3)}}{\partial y}-\frac{\partial F_{n, l}^{(2)}}{\partial z}\right)=m_{0} H_{n, l}^{(1)}, \\
\left(\frac{\partial G_{n, l}^{(3)}}{\partial z}+\frac{\partial G_{n, l}^{(2)}}{\partial y}+\frac{\partial G_{n, l}^{(1)}}{\partial x}\right)=m_{0} \phi_{n, l}
\end{gathered}
$$




$$
\begin{gathered}
E_{n, l} G_{n, l}^{(1)}+i\left(\frac{\partial H_{n, l}^{(3)}}{\partial y}-\frac{\partial H_{n, l}^{(2)}}{\partial z}\right)=m_{0} F_{n, l}^{(1)}, \\
E_{n, l} G_{n, l}^{(2)}+i\left(\frac{\partial H_{n, l}^{(1)}}{\partial z}-\frac{\partial H_{n, l}^{(3)}}{\partial x}\right)=m_{0} F_{n, l}^{(2)}, \\
E_{n, l} G_{n, l}^{(3)}+i\left(\frac{\partial H_{n, l}^{(2)}}{\partial x}-\frac{\partial H_{n, l}^{(1)}}{\partial y}\right)=m_{0} F_{n, l}^{(3)}, \\
\left(E_{n, l}-V(r)\right) F_{n, l}^{(1)}+\frac{\partial \varphi}{\partial x}=m_{0} G_{n, l}^{(1)}, \\
\left(E_{n, l}-V(r)\right) F_{n, l}^{(2)}+\frac{\partial \varphi}{\partial y}=m_{0} G_{n, l}^{(2)}, \\
\left(E_{n, l}-V(r)\right) F_{n, l}^{(3)}+\frac{\partial \varphi}{\partial z}=m_{0} G_{n, l}^{(3)} .
\end{gathered}
$$

Combining the above equations, we have

$$
\begin{gathered}
i \nabla \times \vec{F}=m_{0} \vec{H}, \\
\nabla \cdot \vec{G}=m_{0} \phi_{n, l}, \\
E_{n, l} \vec{G}+i \nabla \times \vec{H}=m_{0} \vec{F}, \\
\left(E_{n, l}-V(r)\right) \vec{F}+\nabla \phi_{n, l}=m_{0} \vec{G} .
\end{gathered}
$$

In obtaining the last result as follows, we combine the above equations:

$$
\begin{gathered}
\left(E_{n, l}\left(E_{n, l}-V(r)\right)-m_{0}^{2}\right) \vec{F}-\nabla \times(\nabla \times \vec{F})+\nabla(\nabla \cdot \vec{F})=0, \\
\nabla \times(\nabla \times \vec{F})=\nabla(\nabla \cdot \vec{F})-\nabla^{2} \vec{F} .
\end{gathered}
$$

Then,

$$
\left(E_{n, l}\left(E_{n, l}-V(r)\right)-m_{0}^{2}\right) \vec{F}+\nabla^{2} \vec{F}=0
$$

Moreover,

$$
\nabla^{2}=\frac{\partial^{2}}{\partial r^{2}}+\frac{2}{r} \frac{\partial}{\partial r}-\frac{L^{2}}{r^{2}}
$$


So we have,

$$
\left(\frac{d^{2}}{d r^{2}}+\frac{2}{r} \frac{d}{d r}+E_{n, l}^{2}-E_{n, l} V(r)-m_{0}^{2}-\frac{l(l+1)}{r^{2}}\right) \vec{F}=0 .
$$

Thus, if we suppose that $V(r)=0$, this equation reduces to $P^{2}=E_{n, l}^{2}-m_{0}^{2}$.

\section{Exact Solutions of DKP Equation under Coulomb Potential}

Now for deriving eigenvalues of energy and wave functions of (3.20), we have

$$
\frac{d^{2} \vec{F}}{d r^{2}}+\frac{2}{r} \frac{d \vec{F}}{d r}+\frac{1}{r^{2}}\left(E_{n, l}^{2} r^{2}-E_{n, l} V_{0} r-m_{0}^{2} r^{2}-l(l+1)\right) \vec{F}=0
$$

We determine the energy eigenvalues from (4.2) as follows:

$$
(2 n+1) \sqrt{m_{0}^{2}-E_{n, l}^{2}}+E_{n, l} V_{0}+2 \sqrt{\left(\frac{1}{4}+l(l+1)\right)\left(m_{0}^{2}-E_{n, l}^{2}\right)}=0
$$

So

$$
\begin{gathered}
E_{n, l}= \pm \frac{2 m_{0}(n+l+1)}{\sqrt{V_{0}^{2}+4(n+l+1)^{2}}}, \\
\varphi_{n, l}^{(2)}(\vec{r})=N_{n, l} r^{l} e^{-\sqrt{m_{0}^{2}-E_{n, l}^{2} r} L_{n}^{2 l+1}\left(2 \sqrt{m_{0}^{2}-E_{n, l}^{2}} r\right) .}
\end{gathered}
$$

We have plotted wave function versus $r$ in Figure 1. The energy eigenvalues for different $l$ are reported in Table 1 to give a better view of the obtained results. Also, we have displayed the energy eigenvalues versus $V_{0}$ in Figure 2. We can now demonstrate that the spectra given in Table 1 present the pattern appearing in supersymmetry quantum mechanics [32] where the levels of energy $E_{n, l}=E_{n+1, l-1}$ are degenerate.

\section{Exact Solutions of DKP Equation under Harmonic Oscillator Potential}

In this section, we study (3.20) with harmonic oscillator potential,

$$
\left(\frac{d^{2}}{d r^{2}}+\frac{2}{r} \frac{d}{d r}+E_{n, l}^{2}-E_{n, l} \alpha r^{2}-m_{0}^{2}-\frac{l(l+1)}{r^{2}}\right) \vec{F}=0
$$




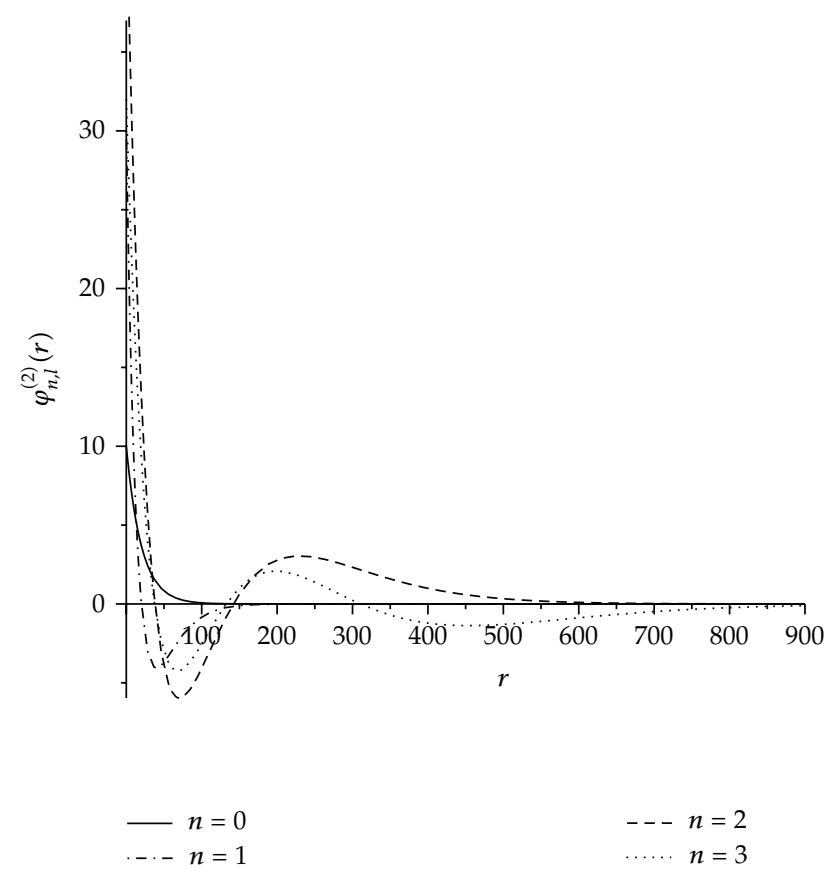

Figure 1: Wave functions for coulomb interaction versus $r$ for $\left(m_{0}=1, V_{0}=-0.1\right.$, and $\left.l=0\right)$.

Table 1: Energy eigenvalues for coulomb interaction with $\left(m_{0}=1, V_{0}=-0.1\right)$.

\begin{tabular}{lccccccc}
\hline$|n, l\rangle$ & $E_{n, l}$ & $|n, l\rangle$ & $E_{n, l}$ & $|n, l\rangle$ & $E_{n, l}$ & $|n, l\rangle$ & $E_{n, l}$ \\
\hline$|0,0\rangle$ & 0.998752 & $|0,1\rangle$ & 0.999688 & $|0,2\rangle$ & 0.999861 & $|0,3\rangle$ & 0.999922 \\
$|1,0\rangle$ & 0.999688 & $|1,1\rangle$ & 0.999861 & $|1,2\rangle$ & 0.999922 & $|1,3\rangle$ & 0.999950 \\
$|2,0\rangle$ & 0.999861 & $|2,1\rangle$ & 0.999922 & $|2,2\rangle$ & 0.999950 & $|2,3\rangle$ & 0.999965 \\
$|3,0\rangle$ & 0.999922 & $|3,1\rangle$ & 0.999950 & $|3,2\rangle$ & 0.999965 & $|3,3\rangle$ & 0.999974 \\
$|4,0\rangle$ & 0.999950 & $|4,1\rangle$ & 0.999965 & $|4,2\rangle$ & 0.999974 & $|4,3\rangle$ & 0.999980 \\
$|5,0\rangle$ & 0.999965 & $|5,1\rangle$ & 0.999974 & $|5,2\rangle$ & 0.999980 & $|5,3\rangle$ & 0.999985 \\
$|6,0\rangle$ & 0.999974 & $|6,1\rangle$ & 0.999980 & $|6,2\rangle$ & 0.999985 & $|6,3\rangle$ & 0.999988 \\
$|7,0\rangle$ & 0.999980 & $|7,1\rangle$ & 0.999985 & $|7,2\rangle$ & 0.999988 & $|7,3\rangle$ & 0.999990 \\
$|8,0\rangle$ & 0.999985 & $|8,1\rangle$ & 0.999988 & $|8,2\rangle$ & 0.999990 & $|8,3\rangle$ & 0.999991 \\
\hline
\end{tabular}

So the energy eigenvalues can be derived from the below equation:

$$
\left(n+\frac{3}{4}+\frac{l}{2}\right) \sqrt{\alpha E_{n, l}}+\frac{m_{0}^{2}-E_{n, l}^{2}}{4}=0,
$$

and the wave function is

$$
\varphi_{n, l}^{(2)}=N_{n, l} r^{l} e^{-\left(\sqrt{\alpha E_{n, l}} / 2\right) r^{2}} L_{n}^{(l+1 / 2)}\left(\sqrt{\alpha E_{n, l}} r^{2}\right) .
$$




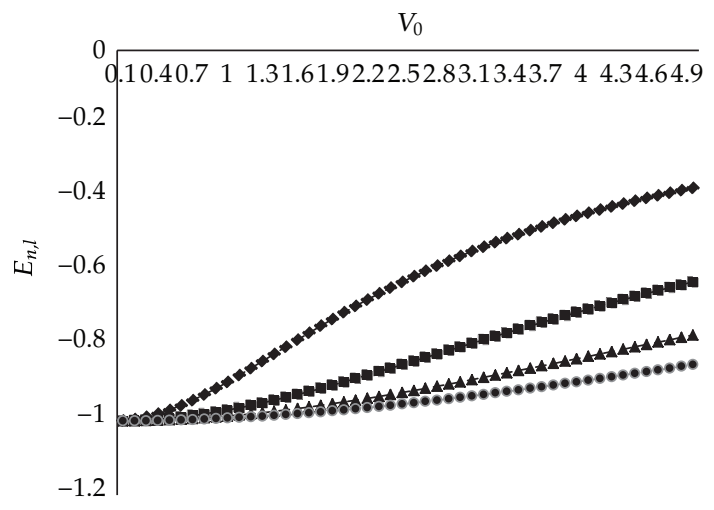

$$
\begin{aligned}
-n=0, L=0 & -n=2, L=0 \\
-n=1, L=0 & -n=3, L=0
\end{aligned}
$$

Figure 2: $E_{n, l}$ versus $V_{0}$.

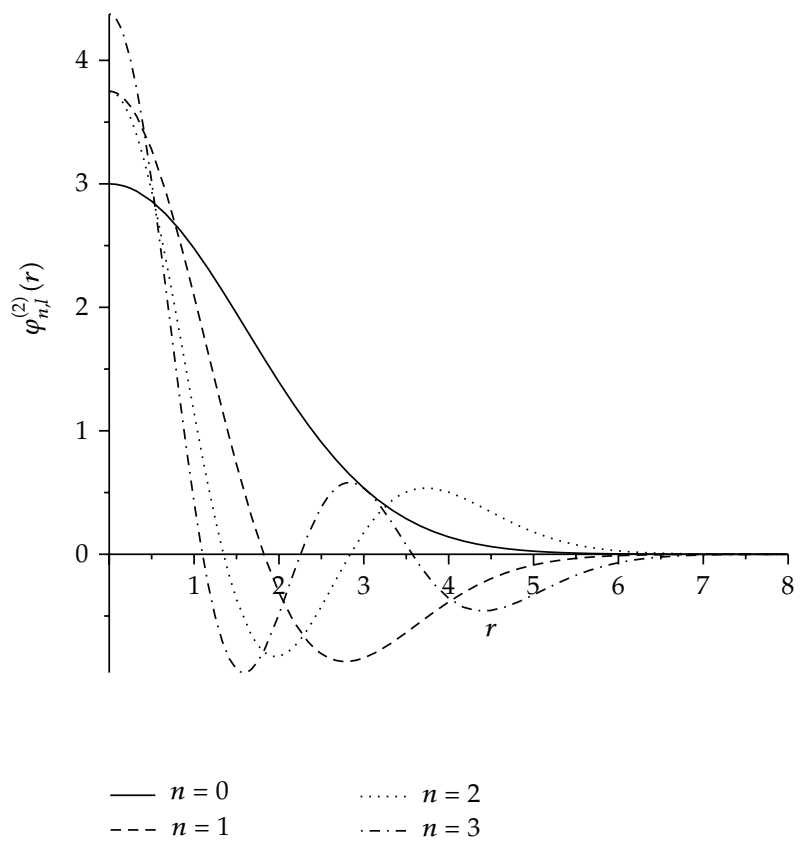

Figure 3: Wave functions for harmonic oscillator potential versus $r$ for $\left(m_{0}=1, \alpha=0.1\right.$, and $\left.l=0\right)$.

We have plotted wave functions versus $r$ in Figure 3. The energy eigenvalues for different $l$ are shown in Table 2, and in Figure 4 we have plotted energy eigenvalues versus $\alpha$.

\section{Conclusion}

We have investigated DKP equation in the presence of coulomb and harmonic oscillator potentials in three-dimensional of space-time for spin-one particles. Thus, we have derived 


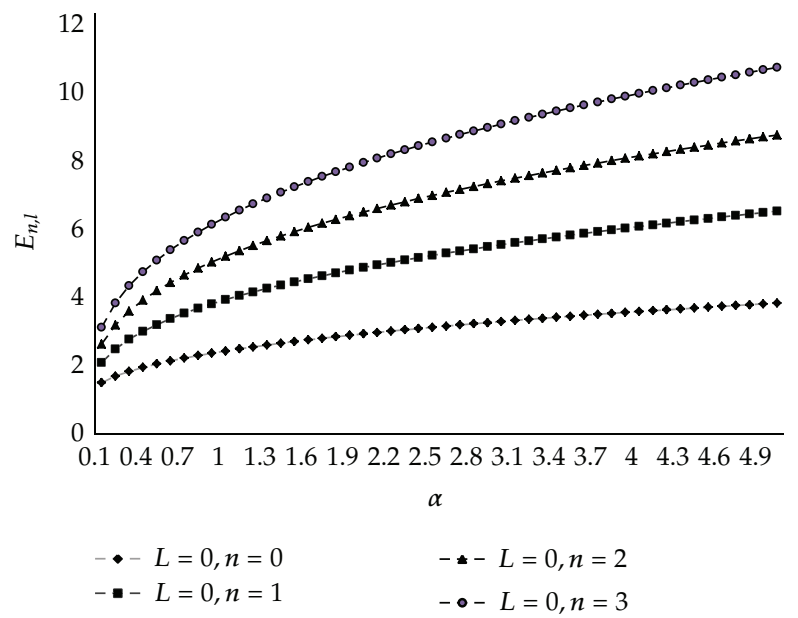

Figure 4: Energy eigenvalues versus $\alpha$.

Table 2: Energy eigenvalues for harmonic oscillator with $\left(m_{0}=1, \alpha=0.1\right)$.

\begin{tabular}{cccccccc}
\hline$|n, l\rangle$ & $E_{n, l}$ & $|n, l\rangle$ & $E_{n, l}$ & $|n, l\rangle$ & $E_{n, l}$ & $|n, l\rangle$ & $E_{n, l}$ \\
\hline$|0,0\rangle$ & 1.465801 & $|0,1\rangle$ & 1.760006 & $|0,2\rangle$ & 2.040014 & $|0,3\rangle$ & 2.307153 \\
$|1,0\rangle$ & 2.040014 & $|1,1\rangle$ & 2.307153 & $|1,2\rangle$ & 2.562975 & $|1,3\rangle$ & 2.808890 \\
$|2,0\rangle$ & 2.562975 & $|2,1\rangle$ & 2.808890 & $|2,2\rangle$ & 3.046099 & $|2,3\rangle$ & 3.275606 \\
$|3,0\rangle$ & 3.046099 & $|3,1\rangle$ & 3.275606 & $|3,2\rangle$ & 3.498248 & $|3,3\rangle$ & 3.714727 \\
$|4,0\rangle$ & 3.498248 & $|4,1\rangle$ & 3.714727 & $|4,2\rangle$ & 3.925637 & $|4,3\rangle$ & 4.131483 \\
$|5,0\rangle$ & 3.925637 & $|5,1\rangle$ & 4.131483 & $|5,2\rangle$ & 4.332698 & $|5,3\rangle$ & 4.529657 \\
$|6,0\rangle$ & 4.332698 & $|6,1\rangle$ & 4.529657 & $|6,2\rangle$ & 4.722685 & $|6,3\rangle$ & 4.912070 \\
$|7,0\rangle$ & 4.722685 & $|7,1\rangle$ & 4.912070 & $|7,2\rangle$ & 5.098064 & $|7,3\rangle$ & 5.280890 \\
$|8,0\rangle$ & 5.098064 & $|8,1\rangle$ & 5.280890 & $|8,2\rangle$ & 5.460749 & $|8,3\rangle$ & 5.637821 \\
\hline
\end{tabular}

energy eigenvalues and wavefunctions where we have plotted the wavefunctions versus $r$ also the energy eigenvalues have been determined in Tables 1 and 2. In effect with increasing the quantum numbers, the values of energy increased. In order to describe the behavior of the energy versus $V_{0}$ and $\alpha$, we have displayed in Figures 2 and 4 that with decreasing of the $\alpha$ and $V_{0}$ the values of energy tend to one point. Furthermore we have discussed the solutions of the DKP equation. Hence, our results are useful in the study of relativistic spin-one particles.

\section{Acknowledgment}

The authors wish to give our sincere gratitude to the referees for their instructive comments and careful reading of the paper.

\section{References}

[1] N. Kemmer, "Quantum theory of Einstein-Bose particles and nuclear interaction," Proceedings of the Royal Society A, vol. 166, pp. 127-153, 1938. 
[2] R. J. Duffin, "On the characteristic matrices of covariant systems," Physical Review, vol. 54, no. 12, p. 1114, 1938.

[3] G. Petiau, University of Paris thesis, Published in Académie Royale de Médecine de Belgique, Classe des Sciences, Mémoires in $8^{\circ}, 16,2,1936$.

[4] V. Gribov, "QCD at large and short distances (annotated version)," European Physical Journal C, vol. 10, no. 1, pp. 71-90, 1999.

[5] I. V. Kanatchikov, "De Donder-Weyl theory and a hypercomplex extension of quantum mechanics to field theory," Reports on Mathematical Physics, vol. 43, no. 1-2, pp. 157-170, 1999.

[6] V. M. Red'kov, "Generally relativistical Tetrode-Weyl-Fock-Ivanenko formalism and behaviour of quantum-mechanical particles of spin 1/2 in the Abelian monopole field," 26 pages, 2008, http://arxiv.org/pdf/quant-ph/9812007.pdf .

[7] J. T. Lunardi, B. M. Pimentel, and R. G. Teixeira, "Duffin-Kemmer-Petiau equation in Riemannian space-times," in Geometrical Aspects of Quantum Fields, pp. 111-127, World Scientific, River Edge, NJ, USA, 2001.

[8] S. Ait-Tahar, J. S. Al-Khalili, and Y. Nedjadi, "A relativistic model for $\alpha$-nucleus elastic scattering," Nuclear Physics A, vol. 589, pp. 307-319, 1995.

[9] I. V. Kanatchikov, "On the Duffin-Kemmer-Petiau formulation of the covariant Hamiltonian dynamics in field theory," Reports on Mathematical Physics, vol. 46, no. 1-2, pp. 107-112, 2000.

[10] J. T. Lunardi, B. M. Pimentel, R. G. Teixeira, and J. S. Valverde, "Remarks on Duffin-Kemmer-Petiau theory and gauge invariance," Physics Letters A, vol. 268, no. 3, pp. 165-173, 2000.

[11] J. T. Lunardi, B. M. Pimentel, J. S. Valverde, and L. A. Manzoni, "Duffin-Kemmer-Petiau theory in the causal approach," International Journal of Modern Physics A, vol. 17, no. 2, pp. 205-227, 2002.

[12] N. Debergh, J. Ndimubandi, and D. Strivay, "On relativistic scalar and vector mesons with harmonic oscillatorlike interactions," Zeitschrift für Physik C, vol. 56, p. 421, 1992.

[13] Y. Nedjadi and R. C. Barret, "The Duffin-Kemmer-Petiau oscillator," Journal of Physics A, vol. 27, p. 4301, 1994.

[14] G. Guo, C. Long, Z. Yang et al., "DKP oscillator in noncommutative phase space," Canadian Journal of Physics, vol. 87, pp. 989-993, 2009.

[15] I. Boztosun, M. Karakoc, F. Yasuk, and A. Durmus, "Asymptotic iteration method solutions to the relativistic Duffin-Kemmer-Petiau equation," Journal of Mathematical Physics, vol. 47, no. 6, p. 062301, 11, 2006.

[16] F. Yasuk, M. Karakoc, and I. Boztosun, "The relativistic Duffin-Kemmer-Petiau sexticoscillator," Physica Scripta, vol. 78, Article ID 045010, 2008.

[17] M. Falek and M. Merad, "DKP oscillator in a noncommutative space," Communications in Theoretical Physics, vol. 50, no. 3, pp. 587-592, 2008.

[18] S. Zarrinkamar, A. A. Rajabi, H. Rahimov, and H. Hassanabadi, "DKP equation under a vector Hulthén-type potential: an approximate solution," Modern Physics Letters A, vol. 26, no. 22, pp. 1621$1629,2011$.

[19] H. Hassanabadi, B. H. Yazarloo, S. Zarrinkamar, and A. A. Rajabi, “Duffin-Kemmer-Petiau equation under a scalar Coulomb interaction," Physical Review C, vol. 84, Article ID 064003, 2011.

[20] R. Oudi, S. Hassanabadi, A. A. Rajabi, and H. Hassanabadi, "Approximate bound state solutions of DKP equation for any J state in the presence of Woods-Saxon Potential," Communications in Theoretical Physics, vol. 57, pp. 15-18, 2012.

[21] H. Hassanabadi, S. F. Forouhandeh, H. Rahimov, S. Zarrinkamar, and B. H. Yazarloo, "DuffinKemmer-Petiau equation under a scalar and vector Hulthen potential; an ansatz solution to the corresponding Heun equation," Canadian Journal of Physics, vol. 90, no. 3, pp. 299-304, 2012.

[22] A. Boumali, "Particule de spin-1 dans un potentiel d'Aharonov-Bohm," Canadian Journal of Physics, vol. 85, pp. 1417-1429, 2007.

[23] A. Boumali, "On the eigensolutions of the one-dimensional Duffin-Kemmer-Petiau oscillator," Journal of Mathematical Physics, vol. 49, no. 2, Article ID 022302, 2008.

[24] M. Merad and S. Bensaid, "Wave functions for a Duffin-Kemmer-Petiau particle in a time-dependent potential," Journal of Mathematical Physics, vol. 48, no. 7, Article ID 073515, 2007.

[25] Y. Kasri and L. Chetouani, "Energy spectrum of the relativistic Duffin-Kemmer-Petiau equation," International Journal of Theoretical Physics, vol. 47, no. 9, pp. 2249-2258, 2008.

[26] A. Boumali and L. Chetouani, "Exact solutions of the Kemmer equation for a Dirac oscillator," Physics Letters A, vol. 346, no. 4, pp. 261-268, 2005. 
[27] P. Ghose, M. K. Samal, and A. Datta, "Klein paradox for bosons," Physics Letters A, vol. 315, no. 1-2, pp. 23-27, 2003.

[28] H. Hassanabadi, Z. Molaee, M. Ghominejad, and S. Zarrinkamar, "Duffin-Kemmer-Petiau equation with a hyperbolical potential in (2+1) dimensions for spin-one particles," Few-Body Systems. In press.

[29] Z. Molaee, M. Ghominejad, H. Hassanabadi, and S. Zarrinkamar, "S-wave solutions of spin-one DKP equation for a deformed Hulthén potential in (1+3) dimensions," European Physical Journal Plus, vol. 127, p. 116, 2012.

[30] Y. Chargui, A. Trabelsi, and L. Chetouani, "Bound-states of the (1+1)-dimensional DKP equation with a pseudoscalar linear plus Coulomb-like potential," Physics Letters A, vol. 374, no. 29, pp. 2907-2913, 2010.

[31] R. E. Kozack, B. C. Clark, S. Hama, V. K. Mishra, R. L. Mercer, and L. Ray, "Spin-one Kemmer-DuffinPetiau equations and intermediate-energy deuteron-nucleus scattering," Physical Review C, vol. 40, no. 5, pp. 2181-2194, 1989.

[32] F. Cooper, A. Khare, and U. Sukhatme, "Supersymmetry and quantum mechanics," Physics Reports, vol. 251, no. 5-6, pp. 267-385, 1995. 

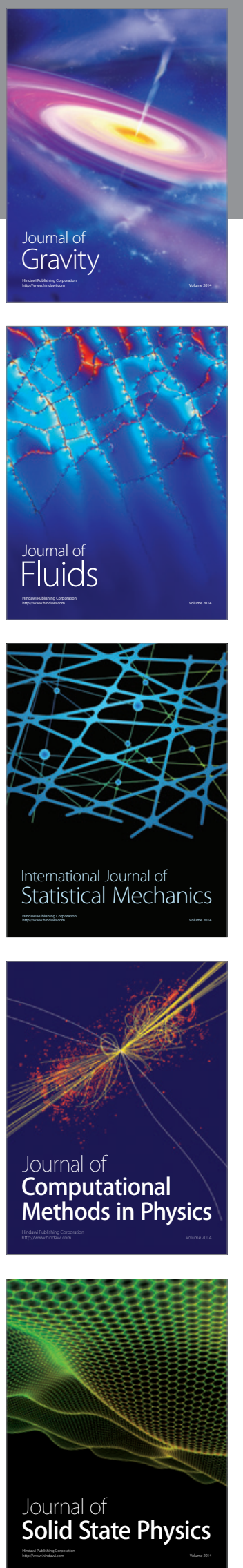

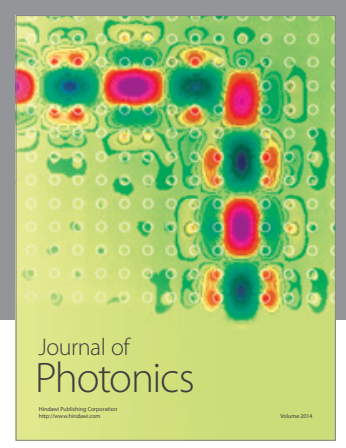

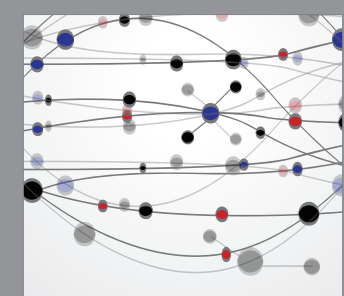

The Scientific World Journal
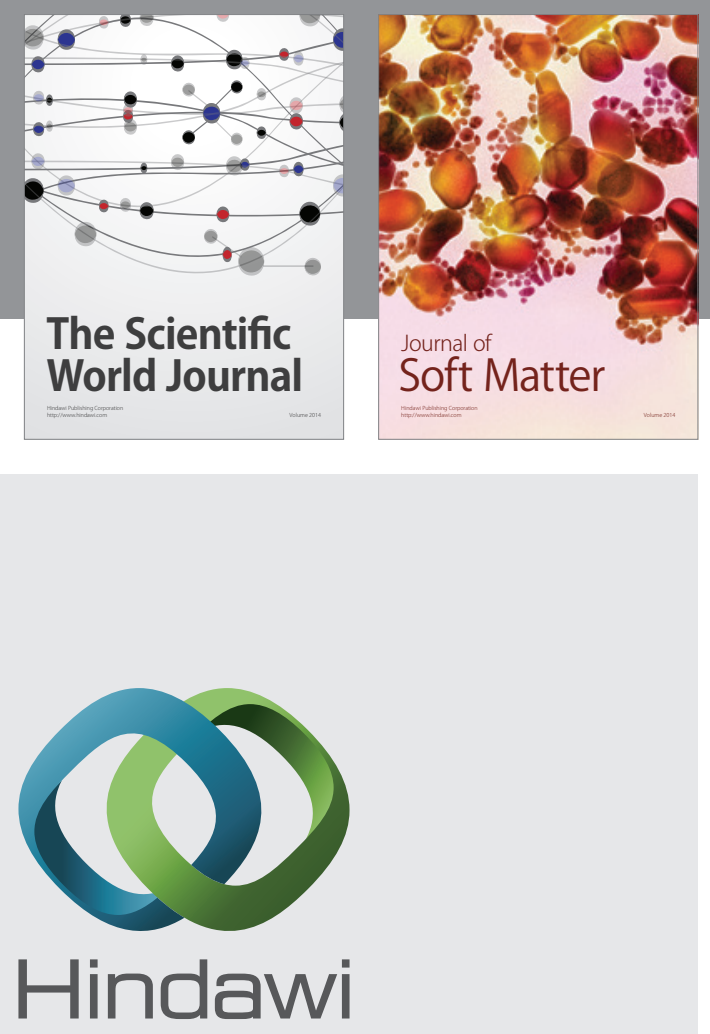

Submit your manuscripts at

http://www.hindawi.com
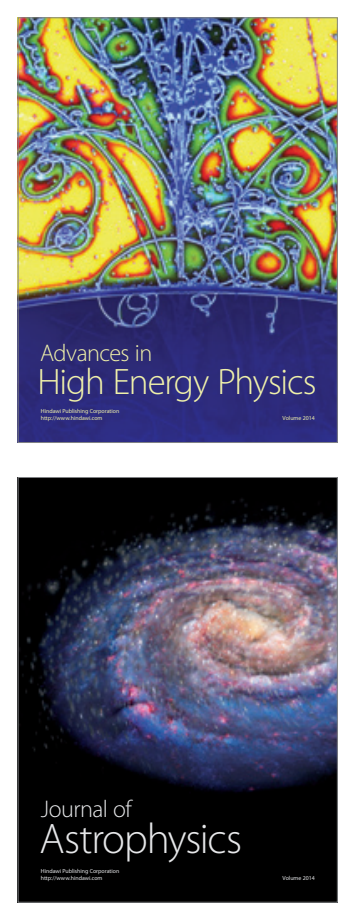
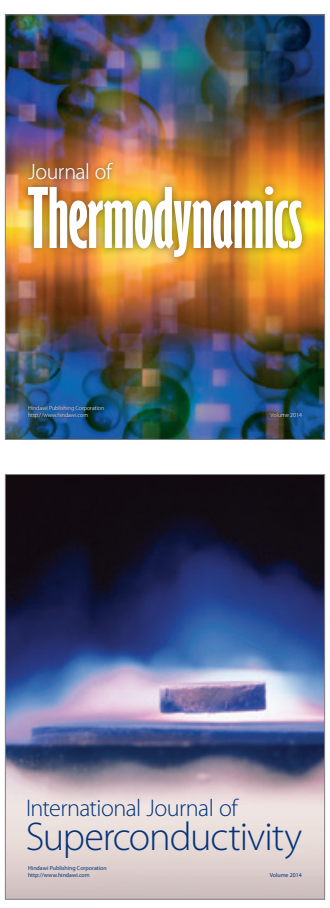
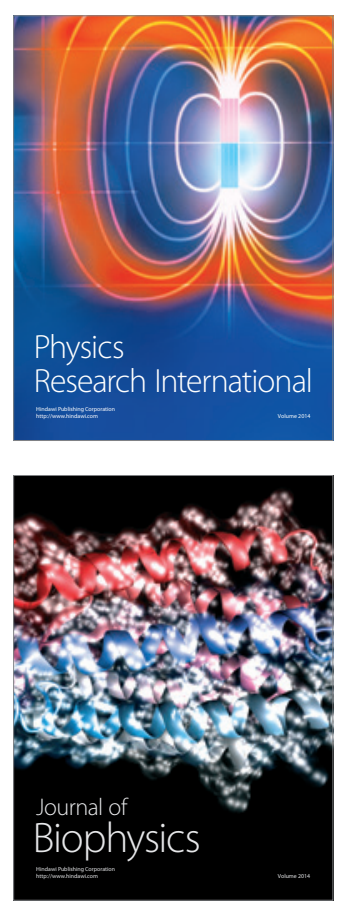
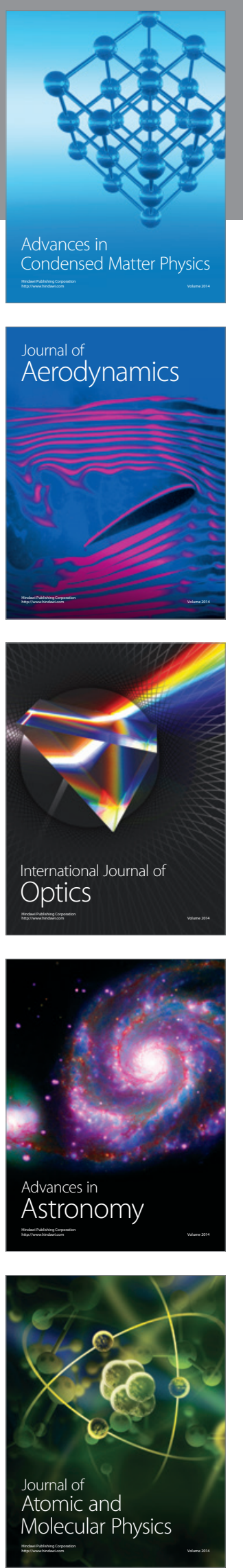